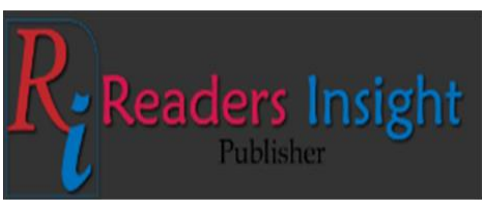

\title{
Dietary Habits and Blood Pressure Control Among Established Hypertensive Patients in Balochistan.
}

\author{
Noor ul Hassan ${ }^{1}$, Farhat Abbas Bokhari ${ }^{1}$, Tahir Hameed ${ }^{* 1}$, Arshad Fayyaz ${ }^{1,2}$, Imran Khan ${ }^{2}$, Fahad \\ Saleem $^{3}$, Zarmina Khan Mandokhail ${ }^{1}$ and Maryam Yousaf ${ }^{1}$
}

${ }^{l}$ Centre for Advanced Studies in Vaccinology and Biotechnology (CASVAB), University of Balochistan, Quetta, Pakistan

${ }^{2}$ Provincial drug testing Laboratory, Government of Balochistan, Quetta, Pakistan.

${ }^{3}$ Department of Biochemistry, University of Balochistan, Quetta, Pakistan.

${ }^{*}$ Correspondin author: tahirquetta@hotmail.com

\section{Abstract}

ARTICLE INFORMATION

Evaluation of effects of hypertension and blood pressure are associated with dietary habits and psychological stress on BP control among established hypertensive patients will facilitate researchers, healthcare practitioners in addition policy makers to establish the association among the diet control and psychological outcomes. Moreover, factors leading to dietary changes, management of stress was highlighted. in the nutshell, some healthcare outcomes. The commendation classifies BP into four classes, $(<120 / 80 \mathrm{~mm} \mathrm{Hg})$ normal, $(120-129 /<80 \mathrm{~mm} \mathrm{Hg})$ raised. $(130-139 / 80-89 \mathrm{~mm} \mathrm{Hg})$ stage $1,(\geq 140 / \geq 90 \mathrm{~mm}$ $\mathrm{Hg}$ ), Stage 2 hypertension This classification is intended to help public and clinical and health verdict and to show observational facts telling a rise in CVD danger as increases in BP from normal to high, to stages 1 and 2 of hypertension.

Keywords: Blood Pressure, Systolic, Diastolic, Hypertension
Received: $\quad 05.05 .2019$

Revised: $\quad 29.05 .2019$

Accepted: $\quad 25.06 .2019$

DOI: 10.31580/pjmls.v2i2.995

\section{INTRODUCTION}

Among the chronic diseases, hypertension is one of the foremost intimidations to mankind. Mortality and disability are major risk factors of hypertension. According to recent worldwide estimate, 874 million adults have systolic blood pressure (SBP) of $140 \mathrm{mmHg}$ or above. Hypertension, in association with chronic kidney disease (CKD), cardio vascular disease (CVD), heart failure, stroke andcerebrovascular accident (CVA) becomes only subsequent cause of death in United Stated (3).

Hypertension is well-defined as diastolic blood pressure $\geq$ $90 \mathrm{mmHg}$ and systolic blood pressure $\geq 140 \mathrm{mmHg}$, being on pharmacological treatment for the hypertension. In the past few decades, cardiovascular disease has been considered one of the highest ten leading causes of disability worldwide (4).

The potential studies watched an immediate connection among circulatory strain levels and cardiovascular also cerebrovascular mortality. As indicated by the after effects of worls' over $25 \%$ of grown-up populace (approx. 1 billion) suffering from hypertension. More then $90 \%$ of high BP patients have primary HTN (2). Primary HTN can't be relieved, yet it very may be controlled with suitable pharmacological treatment (counting lifestyle modifications, medications). One of key aspects is of developing of prime HTN is genetic aspects. Such sort of hypertension develop progressively over various year (5). Primary HTN can't be relieved, yet it very may be controlled with suitable pharmacological treatment (counting lifestyle modifications, medications). Primary HTN can't be relieved, yet it very may be controlled with suitable pharmacological treatment (counting lifestyle modifications, medications).

\section{METHODOLOGY OF THE STUDY}

\section{Study design}

The study was designed as a questionnaire based on cross-sectional analysis. The research was carried out at the cardiac ward of Sandman Provincial Hospital Quetta. Balochistan.

\section{Study population, Sampling and inclusion criteria}

The study samples were the subjects with known high blood pressure hostory for the past 06 months and were administered continuous drug which reduces high blood pressure. Hypertension patients diagnosed within 06 months, illitrate and Afghan refugees were not included in the study (6). For collection of data prevalence-based sampling method was applied. Eighteen percent of Pakistani population is stated to be suffering from hypertension and for sample collection the same prevalence rate was used (7).

\section{Data collection Tools}

The demographic associated information (psychological stress and dietary habits)psychological stress scale (PSS) and dietary habit scale (DHS) was used for the assessment respectively. Values of blood pressure (either controlled or not) were taken from the patient records. Both tools are frequently 
used in research of same nature around the globe. Approval to use the questionnaire was taken by the designers (8). However, the tools were translated and validated for internal consistency and validity when tested in a pilot study.

\section{Data analysis}

The data were coded and entered in SPSS v.20.0 for analysis. Both descriptive and inferential statistics was used for data elaboration. The tools were scored as per standards of the developers. Correlation analysis was adopted to establish the association between the study variables. For all analysis $\mathrm{p}<0.05$ was taken as significant.

\section{RESULTS}

Higher NIHSS were associated with elevated maximal blood pressure levels and decreased discharge DBP and MBP levels. Statistically significant results with a $p<0.05$ are shown in bold letters. The HR of mortality by 1SD increased in blood pressure is shown. Statistically significant results with a $\mathrm{p}<0.05$ are shown in bold letters. When blood pressure was used as a continuous variable, only admission DBP and MBP showed a weak negative association with the risk of total death after first

\begin{tabular}{lllllll}
\hline \multicolumn{3}{l}{ Admission } & \multicolumn{2}{l}{ Maximal } & \multicolumn{2}{c}{ Discharge } \\
\cline { 2 - 7 } & HR & $\mathbf{p}$ & HR & $\mathbf{p}$ & HR & $\mathbf{p}$ \\
SBP & 0.994 & 0.11 & 1.004 & 0.24 & 0.992 & 0.29 \\
DBP & 0.986 & $\mathbf{0 . 0 4}$ & 1.010 & 0.14 & 0.980 & 0.07 \\
PP & 0.996 & 0.49 & 1.003 & 0.58 & 1.002 & 0.85 \\
MBP & 0.988 & $\mathbf{0 . 0 4 8}$ & 1.008 & 0.15 & 0.980 & 0.09 \\
\hline
\end{tabular}

ischemic stroke.

Table I. Blood pressure according to stroke severity scale (NIHSS) quartiles on admission

\section{NIHHS}

HR - hazard ratio; SBP - systolic blood pressure; DBP diastolic blood pressure; PP - pulse pressure; MBP - mean blood pressure.

Table 2. All-cause mortality after ischemic stroke by blood pressure levels.

\begin{tabular}{|c|c|c|c|c|c|c|}
\hline & & Q1 (0-1) & Q2 (2-3) & Q3 (4-7) & Q4 ( $\geq 8)$ & $\begin{array}{l}P \text { for } \\
\text { trend }\end{array}$ \\
\hline \multirow[t]{4}{*}{1} & SBP & $153 \pm 23$ & $161 \pm 28$ & $157 \pm 28$ & $161 \pm 32$ & 0.18 \\
\hline & DBP & $86 \pm 13$ & $91 \pm 14$ & $88 \pm 15$ & $90 \pm 18$ & 0.50 \\
\hline & PP & $67 \pm 19$ & $70 \pm 21$ & $68 \pm 19$ & $71 \pm 23$ & 0.18 \\
\hline & MBP & $109 \pm 14$ & $114 \pm 18$ & $111 \pm 18$ & $113 \pm 21$ & 0.28 \\
\hline \multirow[t]{4}{*}{2} & SBP & $162 \pm 24$ & $171 \pm 27$ & $169 \pm 29$ & $177 \pm 29$ & 0.001 \\
\hline & DBP & $91 \pm 12$ & $97 \pm 13$ & $95 \pm 15$ & $99 \pm 17$ & 0.002 \\
\hline & PP & $72 \pm 20$ & $75 \pm 20$ & $74 \pm 21$ & $79 \pm 21$ & 0.03 \\
\hline & MBP & $115 \pm 14$ & $122 \pm 16$ & $119 \pm 19$ & $125 \pm 19$ & 0.001 \\
\hline \multirow[t]{4}{*}{3} & SBP & $135 \pm 14$ & $138 \pm 16$ & $133 \pm 19$ & $135 \pm 19$ & 0.29 \\
\hline & DBP & $80 \pm 9$ & $80 \pm 9$ & $77 \pm 12$ & $77 \pm 12$ & 0.01 \\
\hline & PP & $88 \pm 13$ & $58 \pm 14$ & $56 \pm 15$ & $58 \pm 15$ & 0.49 \\
\hline & MBP & $98 \pm 10$ & $99 \pm 10$ & $95 \pm 13$ & $96 \pm 13$ & 0.03 \\
\hline
\end{tabular}

Stroke severity on admission was assessed using the National Institute of Health Stroke Scale (NIHSS). NIHSS were divided according to quartiles (Q1-Q4), NIHSS values in each quartile are provided (in brackets). Blood pressure values are mean \pm SD. SBP - systolic blood pressure; DBP - diastolic blood pressure; PP - pulse pressure; MBP - mean blood pressure.

\section{DISCUSSION}

Pre-hypertensive youth treatment is effectively done by timely Dietary modificationwhile adults hypertensive and normotensive systolic and diastolic BP can effectively lower by modification in diet. Dietary approaches to stop hypertension (DASH), rich fruits and vegetables, in addition low fat dairy products (9).

Reduced salt i.ntake, increased dietry potassium and calcium consumption, healthy physical activity and weight loss are the recommended treatment of hypertension by nonpharmacological approaches. These approaches cannot serve as primary measures for the prevention or retard commencement of the hypertension in public but measures could theoretically have important beneficial public health consequences (10).

The DASH diet received grade I score according to American Dietetic Association (ADA)'s whereas, olyunsaturated fatty acids (PUFAs), calcium, magnesium and potassium, were graded as II (fair) which are mainly present in fish oil. The defensive effects of other eleven dietary factors herein group, had inadequate evidence. Sodium is only of 06 proposed dietary risk aspects causing hypertension related with a solid evidence base (11)

\section{Psychological stress}

Steady presentation to stress may be more likely connected to ceaseless BP rises and hypertension event, the quality and length of presentation are assumed to be vital determinants of hazard, impacts of intense stressors on blood pressure (BP) have been demonstrated (12). Biopsychosocial pathway is less clear in deciding how stretch lead to disease. Advancement of essential hypertension is majorly contributed by mental stress. The epidemiologic sign of a joining among stretch and increased blood pressure be situated exceptionally persuading (12).

There's no doubt that hypertension encompasses a complex multifactorial etiology, including hereditary components, brokenness in a few biochemical and physiological administrative forms, and life fashion components counting eat less, physical work out and body weight. Psychosocial components must be put inside this broader setting and be portion of the hazard profile of the person. In a few cases, psychosocial variables may play a definitive part whereas in others their impact may be exceptionally restricted (9). The study was conducted to access the effects of dietary habits on development of HTN and To access the effects of phycological stress on development of HTN (11).

\section{References}

1. Twinamasiko, B., Lukenge, E., Nabawanga, S., Nansalire, W., Kobusingye, L., Ruzaaza, G., \& Bajunirwe, F.). Sedentary Lifestyle and Hypertension in a Periurban Area of Mbarara, 
2. South Western Uganda: A Population Based Cross Sectional Survey. International journal of hypertension, 2018.

3. Cifu, A. S., \& Davis, A. M. (2017). Prevention, detection, evaluation, and management of high blood pressure in adults. Jama, 318(21), 2132-2134.

4. Bell, K., Twiggs, J., Olin, B. R., \& Date, I. R. (2015). Hypertension: the silent killer: updated JNC-8 guideline recommendations. Alabama Pharmacy Association, 334, 4222.

5. Whelton, P. K., Carey, R. M., Aronow, W. S., Casey, D. E., Collins, K. J., Himmelfarb, C. D., ... \& MacLaughlin, E. J. (2018). 2017 ACC/AHA/AAPA/ABC/ACPM/AGS/APhA/ASH/ASPC/NMA/PC NA guideline for the prevention, detection, evaluation, and management of high blood pressure in adults: a report of the American College of Cardiology/American Heart Association Task Force on Clinical Practice Guidelines. Journal of the American College of Cardiology, 71(19), e127-e248.

6. Drenjančević-Perić, I., Jelaković, B., Lombard, J. H., Kunert, M. P., Kibel, A., \& Gros, M. (2011). High-salt diet and hypertension: focus on the renin-angiotensin system. Kidney and blood pressure Research, 34(1), 1-11.

7. Günther, A. L., Liese, A. D., Bell, R. A., Dabelea, D., Lawrence, J. M., Rodriguez, B. L., ... \& Mayer-Davis, E. J. (2009). Association between the dietary approaches to hypertension diet and hypertension in youth with diabetes mellitus. Hypertension, 53(1), 6-12.

8. Spruill, T. M. (2010). Chronic psychosocial stress and hypertension. Current hypertension reports, 12(1), 10-16.

9. Linden, W., Lenz, J. W., \& Con, A. H. (2001). Individualized stress management for primary hypertension: a randomized trial. Archives of Internal Medicine, 161(8), 1071-1080.

10. Steptoe, A. (2000). Psychosocial factors in the development of hypertension. Annals of medicine, 32(5), 371-375.

11. Ford, E. S., \& Cooper, R. S. (1991). Risk factors for hypertension in a national cohort study. Hypertension, 18(5), 598-606.

12. Zhao, D., Qi, Y., Zheng, Z., Wang, Y., Zhang, X. Y., Li, H. J., ... \& Liu, J. (2011). Dietary factors associated with hypertension. Nature Reviews Cardiology, 8(8), 456.

13. Saleem.F..Hassali.A.A..\&

Shafie.A.A(2010).Hypertension in pakistan:time to take some serious action.British Journal of General Practice. 